\title{
Queixas urinárias em mulheres com infarto cerebral
}

\author{
Urinary complaining in stroke women
}

\section{Ébe Santos dos Monteiro', Márcia Maria Gimenez', Sissy Veloso Fontes ${ }^{2}$, Márcia Maiumi Fukujima ${ }^{3}$, Gilmar Fernandes do Prado ${ }^{4}$}

\section{RESUMO}

A incontinência urinária é conceituada como perda involuntária de urina, clinicamente demonstrável que cause problema social ou higiênico. Objetivo. Avaliar a prevalência e os sintomas das queixas urinárias em pacientes que sofreram Acidente Vascular Cerebral Isquêmico (AVCI) e a topografia da lesão. Método. Foram avaliadas 91 mulheres com AVCI segundo os questionários: Mini-Mental e o King`s Health Questionnaire no período de março de 2005 a março de 2006, e divididas em dois grupos: o grupo continente e o grupo de mulheres com sintomas vesicais. A topografia da lesão cerebral foi determinada por tomografia computadorizada. Resultado. Das 91 mulheres, 13 foram excluídas por terem sintomas urinários prévios ao AVCI e por déficits cognitivos, resultando para análise 41 pacientes com sintomas vesicais e 37 continentes, apenas 6 mulheres melhoraram espontaneamente. Não houve relação entre os sintomas urinários e a topografia da lesão. Sintomas urinários ocorreram em $45 \%$ das mulheres pós AVCI. A urge-incontinência ocorreu em 28 pacientes $(68,2 \%)$ e a urgência em 24 pacientes $(58,5 \%)$. Conclusão. Os sintomas de maior freqüência foram urge-incontinência e urgência. Não houve associação significante entre a topografia da lesão e os sintomas urinários.

Unitermos. Incontinência Urinária, AVC, Bexiga Urinária.

Citação. Monteiro ES, Gimenez MM, Fontes SV, Fukujima MM, Prado GF. Queixas urinárias em mulheres com infarto cerebral.

Trabalho realizado na Disciplina de Medicina de Urgência e Medicina Baseada em evidência da UNIFESP, São Paulo-SP, Brasil.

1. Fisioterapeuta, Especialista em uroginecologia, Universidade Federal de São Paulo - UNIFESP, São Paulo-SP, Brasil.

2. Fisioterapeuta, Doutora, UNIFESP, São Paulo-SP, Brasil.

3. Neurologista, Doutora, UNIFESP, São Paulo-SP, Brasil.

4. Neurologista, Doutor, Professor Adjunto do Departamento de Medicina e Chefe do Setor Neuro-Sono do Departamento de Neurologia da UNIFESP, São Paulo-SP, Brasil.

\section{SUMMARY}

Urinary incontinence is defined as an involuntary urine loss. It is clinically demonstrable and causes social and hygienic problems. Objective. To determine the prevalence and symptoms of urinary incontinence in stroke women and verify the association with the lesion topography. Method. We evaluated 91 stroke women using the Mini-mental and King `s Health Questionnaire from March 2005 to March 2006. Women with cognitive deficit and previous urinary incontinency were excluded. We divided them into two groups: continent and incontinent. Lesion topography was determined by tomography scan images. Results. Out of the 91 women, 13 were excluded with cognitive deficits or previous urinary incontinency, resulting in 41 incontinent patients and 37 continent, 6 of them got better spontaneously. Urinary symptoms occurred in $45 \%$ of stroke women. We observed $28(68.2 \%)$ that presented urge-incontinence and $24(58.5 \%)$ with urinary urgency. Conclusion. The main symptoms were urge-incontinence and urgency and there was not a significant association between the lesion topography and the urinary symptoms.

Keyword. Urinary Incontinence, Stroke, Bladder Citation. Monteiro ES, Gimenez MM, Fontes SV, Fukujima MM, Prado GF. Urinary complaining in stroke women.

Endereço para correspondência:

Ébe S Monteiro

R Pedro de Toledo, 598

CEP 04039-001, São Paulo-SP, Brasil

E-mail: ebemonteiro@ibest.com.br, sissyfontes@gmail.com, maiumi.unifesp@gmail.com, gilmarunifesp@yahoo.com.br

Artigo Original Recebido em: 03/05/2008 Revisado em: 04/05/2208 a 15/12/2008

Aceito em: 16/12/2008

Conflito de interesses: não 


\section{INTRODUÇÃO}

A incontinência urinária (IU) é conceituada como toda perda involuntária de urina, clinicamente demonstrada, que cause problema social ou higiênico $^{1}$. A causa mais comum para a IU é neurogênica; havendo um mecanismo de neurônios centrais que controlam parte do trato urinário organizado no cérebro e na medula espinhal ${ }^{2}$. Comprometimentos nesse circuito podem resultar em contração não-inibida do músculo detrusor, que resulta em contrações de forma abrupta e involuntária, caracterizado por bexiga hiperativa quando a origem é idiopática e, bexiga hiperativa neurogênica, quando a origem é neurológica ${ }^{3,4}$.

Os circuitos neurológicos envolvidos na micção compreendem quatro alças (Figura 1). Qualquer alteração de um desses mecanismos pode causar problema de esvaziamento vesical ou incontinência urinaria. $\mathrm{O}$ funcionamento adequado de cada um desses circuitos depende da integridade anatômica de todos. O déficit de qualquer alça pode prejudicar a função do sistema ${ }^{5}$.

A incontinência urinária pós Acidente Vascular Cerebral Isquêmico (AVCI) é atribuída a interrupções do circuito neural da micção, que causa mudanças no tônus da bexiga e do esfíncter ${ }^{5}$.

A prevalência da IU após o Acidente Vascular Cerebral (AVC) na população é alta variando de $32 \%$ a $79 \%$. A incontinência urinária é um forte preditor de mortalidade, défictis neurológicos e piora funcional. Estima-se que a prevalência da bexiga hiperativa na população adulta nos Estados Unidos seja de 16,9\% entre mulheres. As perdas urinárias causam grande impacto na qualidade de vida das mulheres, resultando em problemas sociais e higiê$\operatorname{nicos}^{7-12}$.

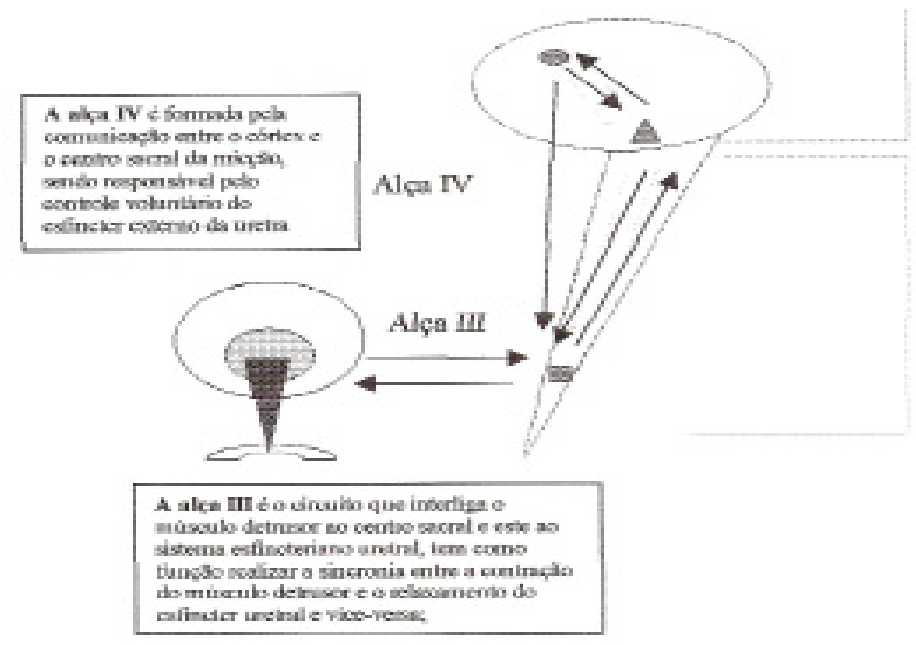

O objetivo desta pesquisa foi verificar a freqüência das queixas urinárias e a topografia da lesão isquêmica do AVCI, nos casos de bexiga hiperativa neurogênica decorrente do AVCI e verificar a associação entre sintomas urinários e a topografia da lesão.

\section{MÉTODO}

Estudo transversal, realizado no período de março de 2005 a março de 2006. Foram recrutadas para a pesquisa mulheres adultas acima de 18 anos com AVCI, diagnóstico clínico confirmado por neuroimagem, sem infecção no trato urinário, tumor vesical e incontinência pré-existente, sem déficit cognitivo (mini-mental < 18) e não gestantes. Foram incluídas 91 mulheres que tiveram AVCI, com idade média de 61,1 anos e foram excluídas $13(14,2 \%)$ por apresentarem déficits cognitivos significantes $(n=8)$ e incontinência urinária prévia $(\mathrm{n}=5)$.

O trabalho foi aprovado pelo Comitê de Ética em Pesquisa da UNIFESP (protocolo 1540/04) e as pacientes assinaram o Termo de Consentimento Informado.

As pacientes foram divididas em dois grupos: mulheres continentes e mulheres incontinentes. Todas as mulheres se encontravam com mais de 7 meses da ocorrência do AVC.
Figura 1

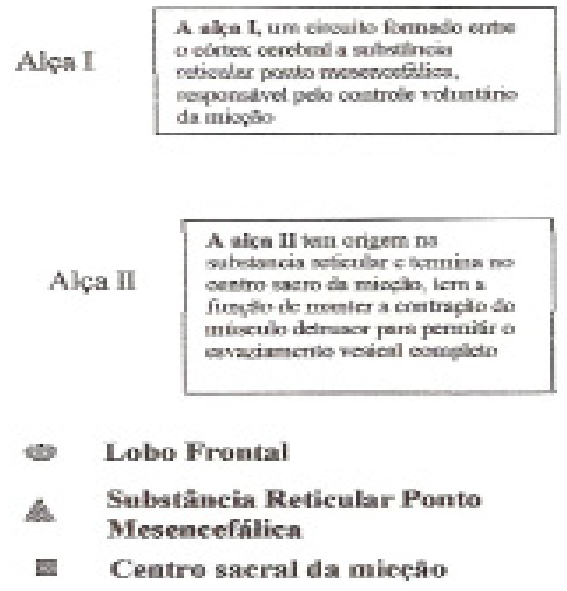




\section{Avaliação}

Todas as mulheres que apresentaram sintomas urinários foram avaliadas segundo o mini-exame do estado mental (MEEM) ${ }^{13}$ para avaliar a cognição, de forma rapida,sendo excluidas as pacientes com MEEM < 18 e o King`s Health Quetionnaire ${ }^{14}$, que para este estudo foi utilizado com ênfase para os dados referentes aos sintomas urinários: freqüência, noctúria, urgência, urge-incontinência, incontinência urinária, enurese e ardência; e dados pessoais: idade e tempo do AVCI. A topografia da lesão foi analisada através do exame de neuroimagem e avaliação médica.

\section{Análise Estatística}

Para a análise estatística utilizou-se o teste do Qui-quadrado para verificar a associação entre a topografia da lesão e a presença de sintomas urinários. Foram usados índices de tendência central (médica); dispersão (desvio padrão) e para a idades que foi comparada entre os grupos utilizou-se o Teste de Mann-Whitney, já os sintomas urinários são descritos em porcentagem.

\section{RESULTADOS}

Das 78 mulheres avaliadas, $37(40,6 \%)$ eram continentes e tinham média de idade de 61,1 anos e $41(45 \%)$ apresentavam disfunções urinárias, com média de idade de 56,5 anos, (com desvio padrão de 11,09), sendo a menor idade 38 anos e a maior idade sendo de 78 anos. Não houve diferença significante em relação às idades entre os grupos $(\mathrm{W}=931)$. Os dados sobre os sintomas urinários da amostra estão na Figura 2.

O hemisfério cerebral lesado não se relacionou com sintomas urinários (Tabela 1). A topografia frontal ocorreu em 73,1\% das pacientes do grupo de mulheres com sintomas urinários, e em apenas 45,9\% do grupo de mulheres sem sintomas urinários.

Figura 2.Frequência dos sintomas miccionais.

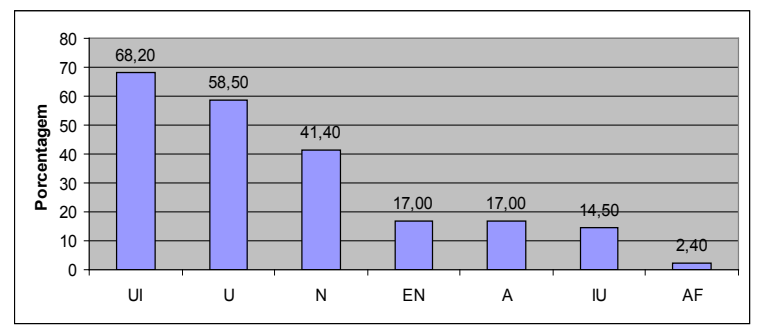

UI=urge incontinência; U=urgência; $\mathrm{N}=$ noctúria; $\mathrm{EN}=$ =nurese noturna; $\mathrm{A}=$ ardência; $\mathrm{IU}=$ incontinência utinária; $\mathrm{AF}=$ aumento da frequênica.
Dentre o grupo que apresentava sintomas urinários, 24 tinham lesão em mais de uma área. As 6 mulheres que melhoraram espontaneamente os sintomas urinários apresentaram lesões em diferentes áreas, sendo 1 região parietal, 3 em área frontal, 1 em parieto-temporal e 1 em região occipital (Tabela 2).

Tabela 1. Topografia da lesão segundo disfunção vesicoesfincteriana.

\begin{tabular}{|l|l|l|l|l|}
\hline \multirow{2}{*}{ Topografia } & \multicolumn{2}{|l|}{ Continente } & \multicolumn{2}{l|}{ Sintomas Urinários } \\
\cline { 2 - 5 } & $\mathrm{n}$ & $\%$ & $\mathrm{n}$ & $\%$ \\
\hline Direito & 17 & 45,9 & 13 & 35,1 \\
\hline Esquerdo & 18 & 48,6 & 25 & 67,6 \\
\hline Tronco & 2 & 5,4 & 3 & 8,1 \\
\hline $\mathrm{p}=0,08$
\end{tabular}

\section{DISCUSSÃO}

Como os sintomas urinários em pacientes com AVCI são pouco estudados, este estudo procurou verificar a freqüência dos sintomas urinários nesses pacientes e correlacioná-los com a topografia da lesão.

Um dos principais sintomas da bexiga hiperativa é a urgência miccional, que tem um grande impacto sobre a qualidade de vida em relação a outros sintomas urinários, formando um círculo vicioso de ansiedade e sofrimento relacionado à possível perda de urina ${ }^{15}$. Sintomas da bexiga hiperativa tem impacto na qualidade de vida de mulheres com e sem incontinência, influenciando negativamente em sua atividades diárias ${ }^{16}$.

Os sintomas de bexiga hiperativa neurogênica não têm relação com as perdas urinárias por esforço, por esta razão é que não faz parte de criterios de analise em pacientes com bexiga hiperativa. Tabela 2. Topografia do infarto lesão cerebral em mulheres segundo sintomas urinários.

Tabela 2. Topografia do infarto lesão cerebral em mulheres segundo sintomas urinários.

\begin{tabular}{|l|l|l|l|l|}
\hline \multirow{2}{*}{ Topografia } & \multicolumn{2}{|l|}{ Sintomas Urinários } & \multicolumn{2}{l|}{ Continente } \\
\cline { 2 - 5 } & $\mathrm{n}(41)$ & $\%$ & $\mathrm{n}(37)$ & $\%$ \\
\hline Frontal & 30 & 73,1 & 17 & 45,9 \\
\hline Temporal & 4 & 9,7 & 4 & 10,8 \\
\hline Tronco & 5 & 12,9 & 2 & 5,4 \\
\hline Occipital & 4 & 9,7 & 5 & 13,5 \\
\hline Parietal & 17 & 41,6 & 17 & 45,9 \\
\hline Cerebelo & 1 & 2,4 & 1 & 2,7 \\
\hline
\end{tabular}

$\mathrm{p}=0,3239$ 
Obtivemos idades menores do que esperávamos (média de 58,8 anos) já que a média encontrada na literatura é em torno de 70 anos, isso implica na prática, termos cada vez mais cedo mulheres procurando ajuda especializada para minimizar os impactos que a disfunção vesico-urinária causa em suas vidas ${ }^{18-20}$

Escolhemos, neste estudo pacientes após 6 meses de lesão, já que sabemos que até neste período ocorre importante recuperação espontânea dos sintomas urinários.Autores como Patel et $\mathrm{al}^{19}$ mostraram que nos primeiros sete dias após AVCI 40\% dos pacientes apresentaram sintomas urinários, esse valor cai para $19 \%$ nos 3 meses seguintes sem tratamento especifico. A incontinência urinária nas quatro primeiras semanas está correlacionado com déficits motores de moderados a severos, influenciando muito no retorno da função até 6 meses pós AVC sugere-se também, um extenso envolvimento cerebrovascular associado à historia de incontinência urinária; pois, $87 \%$ dos pacientes que morreram, no estudo de Vetland 2003, apresentavam incontinência urinária ${ }^{21}$.

Alguns autores relacionam a presença de sintomas urinários com a lesão em região frontal ${ }^{22}$. Apesar de haver mais pacientes com sintomas urinários não foi significante a relação entre topografia da lesão e os sintomas urinários devido ao tamanho da amostra.

A urge-incontinência acompanhada de redução na sensação de enchimento da bexiga foi associada com redução da perfusão global do córtex cerebral, mais especificamente das áreas frontais do cérebro, especialmente no hemisfério direito ${ }^{23}$. Um estudo da literatura mostra que $80 \%$ de pacientes com lesões corticais apresentavam também bexiga hiper ativa, e destes pacientes, $60 \%$ tinham incontinência. As histórias da micturição de 39 pacientes com AVC agudo de tronco mostraram que após três meses do evento, $49 \%$ apresentavam sintomas urinários irritativos e obstrutivos, sendo as mais comuns dificuldades de esvaziamento e aumento na freqüência da noctúria. As imagens de ressonância magnética cerebral destes pacientes ficaram mais concentradas na área dorso lateral da ponte, incluindo o núcleo reticular pontino, a formação reticular adjacente ao núcleo parabraquial e o lócus ceruleus. Estas regiões parecem ser os principais responsáveis pelos tipos supranucleares de disfunção do nervo pudendo; tal fato corresponde ao centro pontino de armazenagem urinária e micção relatados em pesquisas com animais ${ }^{22}$.
Encontramos valores de urgência e urge-incontinência semelhantes ao que vemos na literatura; diferente apenas dos dados vistos em relação ao aumento da freqüência urinária que segundo alguns autores, encontram-se com valores próximos de $60 \%{ }^{24}$; nós encontramos apenas $2,4 \%$. Acreditamos termos encontrado valores menores, já que as mulheres recrutadas não realizaram um diário miccional, (instrumento que consegue quantificar o número exato de vezes que a mulher vai ao banheiro). $\mathrm{O}$ aumento da freqüência urinária, provavelmente não é uma queixa importante para esse grupo de mulheres, sugerindo que outros sintomas como a urgência e a urge-incontinência interfiram muito mais na vida dessas pessoas.

Um estudo realizado na Australia ${ }^{25}$, mostranos o grande impacto que esse problema traz para o sistema de saúde pública, pois naquele ano o tratamento de incontinência urinária foi de $\$ 710,44$ milhõs ou $\$ 387$ por mulher incontinente, somando \$338.47 milhões em custos de tratamento e \$371.97 milhões em custos pessoais. Ainda que este estudo não tenha tratado especificamente da IU pós AVC, seus números dão uma idéia dos custos deste problema para a saúde.

Devem ser feitas novas pesquisas, incluindo o estudo de custo-efetividade de novos tratamentos que vem sendo estudados, como por exemplo a eletroestimulação do nervo tibial posterior; já que este tratamento vem apresentando bons resultados na literatura ${ }^{26}$.

Identificamos como fraquezas do estudo o fato de que não foi avaliada a condição sôcio-econômica-cultural desss pacientes; e também a função motora não foi avaliada.

\section{Conclusão}

A freqüência das queixas urinárias em mulheres com bexiga hiperativa neurogênica foi de $45,05 \%$, e os sintomas mais encontrados foram a urge-incontinência $(68,2 \%)$, a urgência $(58,5 \%)$ e a nocturia $(41,4 \%)$.

Não houve associação estatisticamente significante quando se comparou a topografia da lesão com os sintomas urinários.

Estudos de custo-efetividade são extremamente importantes para melhor direcionarmos o tratamento de bexiga hiperativa. 


\section{Referências Bibliográficas}

1.Abrams P, Van Kerrebroeck P, Chaikin D, Donovan J, Fonda D, Jackson S, et al. The Standardization of terminology of lower urinary tract report from the Standardization Subcommittee of International Continence. Soc Neurourol Urodyn 2002;187:116-26.

2.Rovner E, Gomes C, Trigo-Rocha F, Arap S, Wein J. Evaluation after treatment of the overactive bladder. Rev Hosp Clin 2002;57:1:39-49.

3.International Continence Society Committee on Standardization. The standardization of lower urinary tract function. In: Ostergard DE, Bent $\mathrm{AE}$ (eds). Urogynecology and Urodinamics: Theory and Practice. $3^{\circ}$ ed. Baldimore: Williams, 1991, p.545-62.

4.Gimenez M, Fontes V, Fukujima M. Procedimentos fisioterapêuticos para disfunção vesico-esfincteriana de pacientes com traumatismo raquimedular - revisão narrativa. Rev Neurocienc 2005;13:34-8.

5.Bradley W, Rockward G, Timm GW, Scott F. Inervation of the dertusor muscle and urethra. Urol Clin North Am 1974;1:3-27.

6.Marinkovic SP, Badlani G. Voiding and sexual disfunction after cerobrovascular accidents. J Urol 2001;165:359-70.

7.Gariballa S. Potentially treatable causes of poor outcome in acute stroke patients with urinary incontinence. Acta Neurol Scand 2003;107:336-40.

8.Patel M, Coshall C, Lawrence E. Recovery from poststroke urinary incontinence: associated factors and impact on outcome. Am Geriatr Soc 2001;49:1229-33.

9.Brazier J, Harper R, Jones N, O`Cathain A, Thomas K, Westlake L. Validating the SF-36 Health survey questionaire: new outcome measure for primary care. BMJ 1992;905:160-5.

10.Thakar S, Stanton S. Management of urinary incontinence in women. BMJ 2000;321:1326-31.

11.Kuijk A, Linde H, Limbeek J. Urinary incontinence in strokes patients after admission to a postacute inpatient rehabilitation program. Arch Phys Med Rehabil 2001;82:1407-11.

12.Stewart WF, Herzog R, Wein AJ, Cundiff G, Norton P, Corey R. Prevalence and impact Of Ovaractive Bladder In United States. Results From Noble Program. Neurourol Urodyn 2001;20: 403-422

13.Folstein M, Foltein S, Mchugh P. Mini-Mental State: A practical method for grading the cognitive state of patients for the clinician. J Psychiat Rev 1975;12:189-98.
14.Fonseca ESM, Camargo ALM, Castro RA, Sartori MGF, Fonseca MCM, Lima GR, et al. Validação do questionário de qualidade de vida (King`s Health Questionnaire) em mulheres brasileiras com incontinência urinária. Rev Bras Ginecol Obstet 2005;27:235-42.

15.Symonds T. A review of condition specific instruments to assess the impact of urinary on health related quality of life. Eur Urol 2003;43:21925.

16.Feldner C, Fonseca E, Sartori M, Baracat E, Lima G, Girão M. Síndrome da bexiga hiperativa e qualidade de vida. Femina 2005;33:13-7. 17.Higa R , Lopes MHBM. Fatores associados com a incontinência urinária na mulher. Rev Bras Enferm 2005;58:422-8.

18.Patel M, Coshall C, Lawrence E. Recovery from poststroke urinary incontinence: associated factors and impact on outcome. Am Geriat Soc 2001;49:1229-33.

19.Patel M, Coshall AG, Rudd F, Charles D, Wolfe M. Natural history and effects on 2-Year outcomes of urinary incontinence after stroke. Stroke 2001;32:122-7.

20.Gelber D, Good D, Laven L. Causes of urinary incontinence after acute hemispheric stroke. Stroke 1993;24:378-82.

21.Vetland P. Urinary Continence After A Cerebrovascular Accident. Nurs Stand 2003;17:37-41.

22.Sakakibara R, Uchiyama T, Kuwabara S. Micturitional disturbance after acute hemispheric stroke: analysis of the lesion site by CT and MRI. J Neurol Sci 1996;137:47-56.

23.Griffiths D. Clinical Studies Of Cerebral And Urinary Tract Function In Elderly People With Urinary Incontinence. Behav Brain Res1998;92:151-5.

24.Milson I, Abrams P, Cardozo L. How widespread are the symptoms of an overactive bladder and how are they managed? A populationbased prevalence study. BJU Int 2001;87:760-6.

25.Doran CM, Chiarelli P, Cockburn J. Economic Costs of Urinary Incontinence in Community-Dwelling Australian Women. Med J Aust 2001;174:456-8

26.Amarenco G, Sheikh IS, Even-Schneider A, Raibaut P, DemailleWlodyka S, Parratte B, et al. Urodynamic Effect Of Acute Transcutaneous Posterior Tibial Nerve Stimulation In Overactive Bladder. J Urol 2003;169:2210-5. 